\title{
Directional differentiability of interval-valued functions
}

\author{
Yu-e Bao ${ }^{a, *}, B o Z_{a}^{a}$, Eer-dun Bai ${ }^{b}$ \\ ${ }^{a}$ College of Mathematics, Inner Mongolia University for Nationalities, Tongliao, Inner Mongolia 028043, P. R. China. \\ ${ }^{b}$ College of Computer Science and Technology, Inner Mongolia University for Nationalities, Tongliao, Inner Mongolia \\ 028043, P. R. China.
}

\begin{abstract}
In this paper, by using the generalized Hukuhara difference ( $g H$-difference) of interval numbers, we introduce and study the directional differentiability problem of interval-valued function. Firstly, we put forward the concept of directional differentiability of interval-valued function, discuss the characterizations of directional differentiability of interval-valued function, and propose the sufficient condition of the directional differentiability of interval-valued function. Secondly, we discuss the relations among the directional derivative, derivative and partial derivative of interval-valued function, and prove that the derivative and partial derivative are both special directional derivatives. (C)2016 All rights reserved.
\end{abstract}

Keywords: Interval numbers, interval-valued function, Hausdorff metric, generalized $H$-difference, directional derivative.

2010 MSC: 46G05, 58C25.

\section{Introduction}

Interval analysis (or interval mathematics) is a theory whose basic idea is to use interval variable to calculate instead of point variable. Theory of interval analysis was proposed systematically in 1966 by Moore [5]. From then on, theory and application of interval mathematics developed enormously with the joint effort of many scholars. The interval-valued function and its application receive lots of attention recently [2, 4, 8]. In papers [3, 6, 7] some concepts of differentiability of interval-valued function are introduced, problems about the optimization and differential equation of interval-value are discussed, and some worthy conclusion are obtained.

*Corresponding author

Email address: byebed@163.com (Yu-e Bao) 
Particularly, in order to study the theory of differential equation of interval-valued function better, Stefanini and Bede discussed the generalized differentiability of interval-valued function by means of $g h$-difference, and obtained the concepts of $g h$-derivative and its differentiability of interval-valued function [7]. By using these concepts, Osuna-Gmez et al. [6] proposed the concepts of $g h$-partial derivative and its differentiability of multivariate interval-valued function, discussed the optimization problem about it, and got the optimal conditions which work more widely. Bede and Stefanini discussed the generalized differentiability of fuzzy valued function by means of $g h$-difference, obtained the concepts of $g h$-derivative and its differentiability of fuzzy valued function, and proved its relative properties [1].

In this paper, we put forward the concepts of $g H$-directional derivative and $g H$-directional differentiability of interval-valued functions from a subset of $n$-dimensional Euclidean space $R^{n}$ into interval numbers space $[R]$, study its related properties, and lay a good foundation for the theory of $g H$-directional derivative and differentiability of fuzzy-valued functions from a subset of space into fuzzy numbers space.

\section{Preliminaries}

Let $R$ be the real line. For $\underline{a}, \bar{a} \in R$ and $\underline{a} \leq \bar{a}$, we call $a=[\underline{a}, \bar{a}]$ an interval number, and denote by $[R]$ the set of all interval numbers of the real line $R$, i.e.,

$$
[R]=\{[\underline{a}, \bar{a}] \mid \underline{a}, \bar{a} \in R, \underline{a} \leq \bar{a}\},
$$

and $[R]$ is called interval numbers space. Let $M$ be a nonempty subset of space $R^{n}$ which is an $n$ dimensional Euclidean space. Let $F: M \rightarrow[R]$ be an interval-valued function $F(x)=[\underline{F}(x), \bar{F}(x)]$, where $\underline{F}$ and $\bar{F}$ are real-valued functions defined on $M$ and satisfy $\underline{F}(x) \leq \bar{F}(x)$ for any $x \in M$.

For $a=[\underline{a}, \bar{a}], b=[\underline{b}, \bar{b}] \in R$, we define $a=b$ iff $\underline{a}=\underline{b}$ and $\bar{b}=\bar{b}$. And the Hausdorff metric between interval numbers $a$ and $b$ is defined by

$$
d_{H}(a, b)=\max \{|\underline{a}-\underline{b}|,|\bar{a}-\bar{b}|\} .
$$

Then $\left([R], d_{H}\right)$ is a complete metric space.

Theorem 2.1 ([4]). Let $F: M \rightarrow\left([R], d_{H}\right)$ be an interval-valued function $F(x)=[\underline{F}(x), \bar{F}(x)]$, then $\lim _{x \rightarrow x_{0}} F(x)$ exists if and only if $\lim _{x \rightarrow x_{0}} \underline{F}(x)$ and $\lim _{x \rightarrow x_{0}} \bar{F}(x)$ exist as finite numbers, and

$$
\lim _{x \rightarrow x_{0}} F(x)=\left[\lim _{x \rightarrow x_{0}} \underline{F}(x), \lim _{x \rightarrow x_{0}} \bar{F}(x)\right] .
$$

Definition $2.2([\underline{6}])$. Let $a=[\underline{a}, \bar{a}], b=[\underline{b}, \bar{b}] \in R$. The generalized $H$-difference $(g H$-difference) of two interval numbers and $b$ is defined as follows:

$$
a-{ }_{g} b=c \Leftrightarrow a=b+c \text { or } \quad b=a+(-1) c .
$$

For $a=[\underline{a}, \bar{a}], b=[\underline{b}, \bar{b}] \in R$, the $g H$-difference of two interval numbers $a$ and $b$ always exists and it is equal to (see Proposition 4 in [4])

$$
a-{ }_{g} b=[\min \{\underline{a}-\underline{b}, \bar{a}-\bar{b}\}, \max \{\underline{a}-\underline{b}, \bar{a}-\bar{b}\}] .
$$


Definition $2.3([7])$. Let $x_{0} \in[a, b]$ and $h$ such that $x_{0}+h \in[a, b]$, the $g H$-derivative of the function $F:[a, b] \rightarrow\left([R], d_{H}\right)$ at $x_{0}$ is defined as

$$
F^{\prime}\left(x_{0}\right)=\lim _{h \rightarrow 0} \frac{1}{h}\left[F\left(x_{0}+h\right)-{ }_{g} F\left(x_{0}\right)\right] .
$$

If $F^{\prime}\left(x_{0}\right) \in[R]$, then we say that $F$ is generalized $H$-differentiable ( $g H$-differentiable for short) at $x_{0}$.

Definition $2.4([6])$. Let $F: M \rightarrow\left([R], d_{H}\right)$ be an interval-valued function and $x_{0}=\left(x_{1}^{(0)}, \ldots, x_{n}^{(0)}\right)$ be a fixed element of $M$. We consider the interval-valued function

$$
h_{i}\left(x_{i}\right)=F\left(x_{1}^{(0)}, \ldots, x_{i-1}^{(0)}, x_{i}, x_{i+1}^{(0)}, \ldots, x_{n}^{(0)}\right) .
$$

If $h_{i}$ is $g H$-differentiable at $x_{i}^{(0)}$, then we say that $F$ has the $i$-th partial $g H$-derivative at $x_{0}$ (denoted by $\left.\left(\partial F / \partial x_{i}\right)\left(x_{0}\right)\right)$ and $\left(\partial F / \partial x_{i}\right)\left(x_{0}\right)=h_{i}{ }^{\prime}\left(x_{i}^{(0)}\right) \in[R]$.

Definition $2.5([6])$. Let $F: M \rightarrow\left([R], d_{H}\right)$ be an interval-valued function and let

$$
x_{0}=\left(x_{1}^{(0)}, \ldots, x_{n}^{(0)}\right) \in M
$$

be fixed. We say that $F$ is $g H$-differentiable at $x_{0}$, and we denote by $\nabla F\left(x_{0}\right)$, if all the partial $g H$ derivatives $\left(\partial F / \partial x_{1}\right)\left(x_{0}\right), \ldots,\left(\partial F / \partial x_{n}\right)\left(x_{0}\right)$ exist on some neighborhoods of $x_{0}$ and are continuous at $x_{0}$.

\section{Directional derivatives of interval-valued functions}

In [6], Osuna-Gmez et al. defined the partial $g H$-derivative and $g H$-differentiability of intervalvalued function from a subset of space $R^{n}$ into interval numbers space $[R]$ by using $g H$-difference of interval numbers. In the following, by using $g H$-difference of interval numbers, we defined the $g H$ directional derivative and $g H$-directional differentiability of interval-valued function from a subset of space $R^{n}$ into interval numbers space $[R]$.

In this section, for $y \in R^{n}, y_{e}$ denotes the unit vector of $y$. If $\cos \alpha_{1}, \cos \alpha_{2}, \ldots, \cos \alpha_{n}$ are direction cosines of $y$, then $y_{e}=\left(\cos \alpha_{1}, \cos \alpha_{2}, \ldots, \cos \alpha_{n}\right)$.

Definition 3.1. Let $F: M \rightarrow\left([R], d_{H}\right)$ be an interval-valued function and $x \in M$. If for $y \in R^{n}$, there exists $\delta>0$ such that $x+h y_{e} \in M$ (resp. $\left.x-h y_{e} \in M\right)$ for any real number $h \in(0, \delta)$, and there exists $a^{+} \in[R]$ (resp. $\left.a^{-} \in[R]\right)$ such that

$$
\lim _{h \rightarrow 0^{+}} \frac{1}{h}\left[F\left(x+h y_{e}\right)-{ }_{g} F(x)\right]=a^{+}\left(\lim _{h \rightarrow 0^{+}} \frac{1}{h}\left[F(x)-{ }_{g} F\left(x-h y_{e}\right)\right]=a^{-}\right) .
$$

Then we say $F$ is right (resp. left) $g H$-differentiable in the direction $y$ at $x$, and $a^{+}$(resp. $a^{-}$) the right (resp. left) directional $g H$-derivative of $F$ at $x$ in the direction $y$ and denote

$$
F_{g H}^{+}(x, y)=a^{+}\left(F_{g H}^{-}(x, y)=a^{-}\right)
$$

If $F_{g H}^{+}(x, y)=F_{g H}^{-}(x, y)$, then we say $F$ is $g H$-differentiable in the direction $y$ at $x$, and denote

$$
F_{g H}(x, y)=F_{g H}^{+}(x, y)=F_{g H}^{-}(x, y),
$$

and call $F_{g H}(x, y)$ the directional $g H$-derivative of $F$ at $x$ in the direction $y$. 
Theorem 3.2. Let $F: M \rightarrow\left([R], d_{H}\right)$ be an interval-valued function $F(x)=[\underline{F}(x), \bar{F}(x)]$. If function $F$ is $g H$-differentiable in the direction $y$ at $x$, and there exists $\delta>0$ such that

$$
\begin{aligned}
& \underline{F}\left(x+h y_{e}\right)-\underline{F}(x) \leq \bar{F}\left(x+h y_{e}\right)-\bar{F}(x), \\
& \underline{F}(x)-\underline{F}\left(x-h y_{e}\right) \leq \bar{F}(x)-\bar{F}\left(x-h y_{e}\right)
\end{aligned}
$$

for any $h \in(0, \delta)$, then the directional derivatives of $\bar{F}(x)$ and $\underline{F}(x)$ exist in direction $y$ at $x$, and

$$
F_{g H}(x, y)=[\underline{F}(x, y), \bar{F}(x, y)],
$$

where $\underline{F}(x, y)$ and $\bar{F}(x, y)$ are the direction derivatives of $\underline{F}(x)$ and $\bar{F}(x)$ in direction $y$ at $x$, respectively.

Proof. Necessity.

Let $F(x)=[\underline{F}(x), \bar{F}(x)]$ be $g H$-differentiable in the direction $y$ at $x$, then there exists $\delta>0$ such that $x+h y_{e} \in M, x-h y_{e} \in M$ for any $h \in(0, \delta)$, and there exists $F_{g H}(x, y) \in[R]$ which satisfies

$$
\lim _{h \rightarrow 0^{+}} \frac{F\left(x+h y_{e}\right)-{ }_{g} F(x)}{h}=\lim _{h \rightarrow 0^{+}} \frac{F(x)-{ }_{g} F\left(x-h y_{e}\right)}{h}=F_{g H}(x, y) .
$$

Therefore, we have

$$
\begin{aligned}
& \lim _{h \rightarrow 0^{+}}\left|\frac{F\left(x+h y_{e}\right)-\underline{F}(x)}{h}-\underline{F}_{g H}^{+}(x, y)\right| \\
& =\lim _{h \rightarrow 0^{+}}\left|\frac{\min \left\{\underline{F}\left(x+h y_{e}\right)-\underline{F}(x), \bar{F}\left(x+h y_{e}\right)-\bar{F}(x)\right\}}{h}-\underline{F}_{g H}^{+}(x, y)\right| \\
& \leq \lim _{h \rightarrow 0^{+}} \max \left\{\left|\frac{\min \left\{\underline{F}\left(x+h y_{e}\right)-\underline{F}(x), \bar{F}\left(x+h y_{e}\right)-\bar{F}(x)\right\}}{h}-\underline{F}_{g H}^{+}(x, y)\right|,\right. \\
& \left.\left|\frac{\max \left\{\underline{F}\left(x+h y_{e}\right)-\underline{F}(x), \bar{F}\left(x+h y_{e}\right)-\bar{F}(x)\right\}}{h}-\bar{F}_{g H}^{+}(x, y)\right|\right\} \\
& =d_{H}\left(\frac{F\left(x+h y_{e}\right)-{ }_{g} F(x)}{h}, F_{g H}^{+}(x, y)\right)=0 .
\end{aligned}
$$

We can obtain

$$
\lim _{h \rightarrow 0^{+}}\left|\frac{\underline{F}\left(x+h y_{e}\right)-\underline{F}(x)}{h}-\underline{F}_{g H}^{+}(x, y)\right|=0 .
$$

Therefore, the right derivative of $\underline{F}(x)$ exists in direction $y$ at $x$ and $\underline{F}^{+}(x, y)=\underline{F}_{g H}^{+}(x, y)$. The proof of $\underline{F}^{-}(x, y)=\underline{F}_{a H}^{-}(x, y)$ can be completed with the same argument.

On the other hand, by (3.1) we have

$$
\underline{F}_{g H}^{+}(x, y)=\underline{F}_{g H}^{-}(x, y)=\underline{F}_{g H}(x, y) .
$$

Therefore, the derivative of $\underline{F}(x)$ exists in direction $y$ at $x$, and

$$
\underline{F}_{g H}(x, y)=\underline{F}(x, y) .
$$

Likewise, we can also obtain

$$
\bar{F}_{g H}(x, y)=\bar{F}(x, y) .
$$

So by $(3.2)$ and (3.3), we have

$$
F_{g H}(x, y)=[\underline{F}(x, y), \bar{F}(x, y)] .
$$


Theorem 3.3. Let $F: M \rightarrow\left([R], d_{H}\right)$ be an interval-valued function $F(x)=[\underline{F}(x), \bar{F}(x)]$. If the directional derivative of $\underline{F}(x)$ and $\bar{F}(x)$ exist in direction $y$ at $x_{0}$, then $F(x)$ is $g H$-differentiable in direction $y$ at $x_{0}$ and

$$
F_{g H}\left(x_{0}, y\right)=\left[\min \left\{\underline{F}\left(x_{0}, y\right), \bar{F}\left(x_{0}, y\right)\right\}, \max \left\{\underline{F}\left(x_{0}, y\right), \bar{F}\left(x_{0}, y\right)\right\}\right],
$$

where $\underline{F}\left(x_{0}, y\right)$ and $\bar{F}\left(x_{0}, y\right)$ are the direction derivatives of $\underline{F}(x)$ and $\bar{F}(x)$ in direction $y$ at $x_{0}$, respectively.

Proof.

$$
\begin{aligned}
\lim _{h \rightarrow 0^{+}} \frac{F\left(x_{0}+h y_{e}\right)-{ }_{g} F\left(x_{0}\right)}{h}= & \lim _{h \rightarrow 0^{+}}\left[\frac{1}{h} \min \left\{\underline{F}\left(x_{0}+h y_{e}\right)-\underline{F}\left(x_{0}\right), \bar{F}\left(x_{0}+h y_{e}\right)-\bar{F}\left(x_{0}\right)\right\},\right. \\
& \left.\frac{1}{h} \max \left\{\underline{F}\left(x_{0}+h y_{e}\right)-\underline{F}\left(x_{0}\right), \bar{F}\left(x_{0}+h y_{e}\right)-\bar{F}\left(x_{0}\right)\right\}\right] .
\end{aligned}
$$

On the other hand,

$$
\begin{aligned}
\frac{1}{h} \min \{\underline{F}( & \left.\left.x_{0}+h y_{e}\right)-\underline{F}\left(x_{0}\right), \bar{F}\left(x_{0}+h y_{e}\right)-\bar{F}\left(x_{0}\right)\right\} \\
= & \frac{1}{2 h}\left(\left(\underline{F}\left(x_{0}+h y_{e}\right)-\underline{F}\left(x_{0}\right)\right)+\left(\bar{F}\left(x_{0}+h y_{e}\right)-\bar{F}\left(x_{0}\right)\right)\right. \\
& \left.-\left|\left(\underline{F}\left(x_{0}+h y_{e}\right)-\underline{F}\left(x_{0}\right)\right)-\left(\bar{F}\left(x_{0}+h y_{e}\right)-\bar{F}\left(x_{0}\right)\right)\right|\right) \\
& \frac{1}{h} \max \left\{\underline{F}\left(x_{0}+h y_{e}\right)-\underline{F}\left(x_{0}\right), \bar{F}\left(x_{0}+h y_{e}\right)-\bar{F}\left(x_{0}\right)\right\} \\
= & \frac{1}{2 h}\left(\left(\underline{F}\left(x_{0}+h y_{e}\right)-\underline{F}\left(x_{0}\right)\right)+\left(\bar{F}\left(x_{0}+h y_{e}\right)-\bar{F}\left(x_{0}\right)\right)\right. \\
& -\mid\left(\underline{F}\left(x_{0}+h y_{e}\right)-\underline{F}\left(x_{0}\right)-\left(\bar{F}\left(x_{0}+h y_{e}\right)-\bar{F}\left(x_{0}\right)\right) \mid .\right.
\end{aligned}
$$

Therefore, by Theorem 2.1 we have

$$
\begin{aligned}
\lim _{h \rightarrow 0^{+}} \frac{F\left(x_{0}+h y_{e}\right)-{ }_{g} F\left(x_{0}\right)}{h}= & \lim _{h \rightarrow 0^{+}}\left[\frac{1}{2 h}\left\{\left(\underline{F}^{+}\left(x_{0}, y\right)+\bar{F}^{+}\left(x_{0}, y\right)\right)-\left|\underline{F}^{+}\left(x_{0}, y\right)-\bar{F}^{+}\left(x_{0}, y\right)\right|\right\},\right. \\
& \left.\frac{1}{2 h}\left\{\left(\underline{F}^{+}\left(x_{0}, y\right)+\bar{F}^{+}\left(x_{0}, y\right)\right)+\left|\underline{F}^{+}\left(x_{0}, y\right)-\bar{F}^{+}\left(x_{0}, y\right)\right|\right\}\right] \\
= & {\left[\min \left\{\underline{F}^{+}\left(x_{0}, y\right), \bar{F}^{+}\left(x_{0}, y\right)\right\}, \max \left\{\underline{F}^{+}\left(x_{0}, y\right)+\bar{F}^{+}\left(x_{0}, y\right)\right\}\right] . }
\end{aligned}
$$

Therefore, the right derivative of $F(x)$ exists in direction $y$ at $x$ and

$$
F_{g H}^{+}(x, y)=\left[\min \left\{\underline{F}^{+}\left(x_{0}, y\right), \bar{F}^{+}\left(x_{0}, y\right)\right\}, \max \left\{\underline{F}^{+}\left(x_{0}, y\right), \bar{F}^{+}\left(x_{0}, y\right)\right\}\right] .
$$

Similarly, we can show that the left $g H$-derivative of $F(x)$ exists in direction $y$ at $x$ and

$$
F_{g H}^{-}\left(x_{0}, y\right)=\left[\min \left\{\underline{F}^{-}\left(x_{0}, y\right), \bar{F}^{-}\left(x_{0}, y\right)\right\}, \max \left\{\underline{F}^{-}\left(x_{0}, y\right), \bar{F}^{-}\left(x_{0}, y\right)\right\}\right] .
$$

So by

$$
\underline{F}\left(x_{0}, y\right)=\underline{F}^{-}\left(x_{0}, y\right)=\underline{F}^{+}\left(x_{0}, y\right), \quad \bar{F}\left(x_{0}, y\right)=\bar{F}^{-}\left(x_{0}, y\right)=\bar{F}^{+}\left(x_{0}, y\right),
$$

we can obtain that the function $F(x)$ is differentiable in the direction $y$ at $x_{0}$, and

$$
F_{g H}\left(x_{0}, y\right)=\left[\min \left\{\underline{F}\left(x_{0}, y\right), \bar{F}\left(x_{0}, y\right)\right\}, \max \left\{\underline{F}\left(x_{0}, y\right)+\bar{F}\left(x_{0}, y\right)\right\}\right] .
$$


Example 3.4. Let $M=[-2.2] \times[-2.2]$ and interval-valued function $F: M \rightarrow\left([R], d_{H}\right)$ is given by

$$
F(x)=\left[x_{1}^{2}-x_{2}^{2}, 9-x_{1} x_{2}+x_{2}^{2}\right], \quad x=\left(x_{1}, x_{2}\right) \in M .
$$

Then $F(x)$ is differentiable in the direction $y=(1,3)$ at $x_{0}=(0,1)$, and

$$
F_{g H}\left(x_{0}, y\right)=[-6 / \sqrt{10}, 5 / \sqrt{10}] .
$$

Proof. (1) By using Definition 3.1

$$
\begin{gathered}
\underline{F}(x)=x_{1}^{2}-x_{2}^{2}, \quad \bar{F}(x)=9-x_{1} x_{2}+x_{2}^{2}, \quad y_{e}=(1 / \sqrt{10}, 3 / \sqrt{10}), \\
x_{0}+h y_{e}=(0,1)+h(1 / \sqrt{10}, 3 / \sqrt{10})=(h / \sqrt{10}, 1+3 h / \sqrt{10}), \\
x_{0}-h y_{e}=(0,1)-h(1 / \sqrt{10}, 3 / \sqrt{10})=(-h / \sqrt{10}, 1-3 h / \sqrt{10}), \\
\underline{F}\left(x_{0}+h y_{e}\right)-\underline{F}\left(x_{0}\right)=-6 / \sqrt{10}-8 h^{2} / 10, \\
\bar{F}\left(x_{0}+h y_{e}\right)-\bar{F}\left(x_{0}\right)=5 h / \sqrt{10}+6 h^{2} / 10 .
\end{gathered}
$$

Therefore, we have

$$
\begin{aligned}
F_{g H}^{+}\left(x_{0}, y\right) & =\lim _{h \rightarrow 0^{+}} \frac{F\left(x_{0}+h y_{e}\right)-{ }_{g} F\left(x_{0}\right)}{h} \\
& =\lim _{h \rightarrow 0^{+}} \frac{\left[-6 h / \sqrt{10}-8 h^{2} / 10,5 h / \sqrt{10}+6 h^{2} / 10\right]}{h} \\
& =\lim _{h \rightarrow 0^{+}}[-6 / \sqrt{10}-8 h / 10,5 / \sqrt{10}+6 h / 10]=[-6 / \sqrt{10}, 5 / \sqrt{10}] .
\end{aligned}
$$

On the other hand,

$$
\begin{aligned}
-\underline{F}\left(x_{0}-h y_{e}\right)+\underline{F}\left(x_{0}\right) & =-6 h / \sqrt{10}+8 h^{2} / 10, \\
\bar{F}\left(x_{0}\right)-\bar{F}\left(x_{0}-h y_{e}\right) & =5 h / \sqrt{10}-6 h^{2} / 10 .
\end{aligned}
$$

Therefore, we have

$$
\begin{aligned}
F_{g H}^{-}\left(x_{0}, y\right) & =\lim _{h \rightarrow 0^{+}} \frac{F\left(x_{0}\right)-{ }_{g} F\left(x_{0}-h y_{e}\right)}{h} \\
& =\lim _{h \rightarrow 0^{+}} \frac{\left[-6 h / \sqrt{10}+8 h^{2} / 10,5 h / \sqrt{10}-6 h^{2} / 10\right]}{h}=[-6 / \sqrt{10}, 5 / \sqrt{10}] .
\end{aligned}
$$

So $F_{g H}^{+}\left(x_{0}, y\right)=F_{g H}^{-}\left(x_{0}, y\right)=[-6 / \sqrt{10}, 5 / \sqrt{10}]$. By Definition 3.1, $F(x)$ is $g H$-differentiable in the direction $y$ at $x_{0}$, and

$$
F_{g H}\left(x_{0}, y\right)=[-6 / \sqrt{10}, 5 / \sqrt{10}] .
$$

(2) By using Theorem 3.2

$$
\begin{gathered}
\underline{F}_{x_{1}}\left(x_{0}\right)=\left.2 x_{1}\right|_{(0,1)}=0, \quad \underline{F}_{x_{2}}\left(x_{0}\right)=-\left.2 x_{2}\right|_{(0,1)}=-2, \\
\bar{F}_{x_{1}}\left(x_{0}\right)=-\left.x_{1}\right|_{(0,1)}=0, \quad \bar{F}_{x_{2}}\left(x_{0}\right)=\left.\left(-x_{1}+2 x_{2}\right)\right|_{(0,1)}=2, \\
y_{e}=(\cos \alpha, \cos \beta)=(1 / \sqrt{10}, 3 / \sqrt{10}),
\end{gathered}
$$

where $\cos \alpha, \cos \beta$ are direction cosines of $y=(1,3)$. 
Therefore, we have

$$
\begin{aligned}
& \underline{F}\left(x_{0}, y\right)=\underline{F}_{x_{1}}\left(x_{0}\right) \cos \alpha+\underline{F}_{x_{2}}\left(x_{0}\right) \cos \beta=-6 / \sqrt{10}, \\
& \bar{F}\left(x_{0}, y\right)=\bar{F}_{x_{1}}\left(x_{0}\right) \cos \alpha+\bar{F}_{x_{2}}\left(x_{0}\right) \cos \beta=5 / \sqrt{10} .
\end{aligned}
$$

So by Theorem 3.2 , we have $F(x)$ is $g H$-differentiable in the direction $y$ at $x_{0}$, and

$$
F_{g H}\left(x_{0}, y\right)=[-6 / \sqrt{10}, 5 / \sqrt{10}] .
$$

Theorem 3.5. Let $F:(a, b) \rightarrow\left([R], d_{H}\right)$ be an interval-valued function $F(x)=[\underline{F}(x), \bar{F}(x)]$. Then $F(x)$ is $g H$-differentiable in the direction $y=1$ at $x$ if and only if $F(x)$ is $g H$-differentiable at $x$ and

$$
F^{\prime}(x)=F_{g H}(x, 1) .
$$

Proof. Necessity.

Let $F(x)$ be $g H$-differentiable in the direction $y=1$ at $x$, then there exists $\delta>0$ such that $x+h \in(a, b), x-h \in(a, b)$, for any $h \in(0, \delta)$. There also exists $F_{g H}(x, 1) \in[R]$ such that

$$
\lim _{h \rightarrow 0^{+}} \frac{F(x+h)-{ }_{g} F(x)}{h}=\lim _{h \rightarrow 0^{+}} \frac{F(x+h)-{ }_{g} F(x)}{h}=F_{g H}(x, 1) .
$$

On the other hand, we obtain

$$
\begin{aligned}
-(F(x) & \left.-{ }_{g} F(x-h)\right) \\
& =-[\min \{\underline{F}(x)-\underline{F}(x-h), \bar{F}(x)-\bar{F}(x-h)\}, \max \{\underline{F}(x)-\underline{F}(x-h), \bar{F}(x)-\bar{F}(x-h)\}] \\
& =[-\max \{\underline{F}(x)-\underline{F}(x-h), \bar{F}(x)-\bar{F}(x-h)\},-\min \{\underline{F}(x)-\underline{F}(x-h), \bar{F}(x)-\bar{F}(x-h)\}] \\
& =[\min \{\underline{F}(x-h)-\underline{F}(x), \bar{F}(x-h)-\bar{F}(x)\}, \max \{\underline{F}(x-h)-\underline{F}(x), \bar{F}(x-h)-\bar{F}(x)\}] .
\end{aligned}
$$

Therefore, we have

$$
\lim _{h \rightarrow 0^{+}} \frac{F(x)-{ }_{g} F(x-h)}{h}=\lim _{h \rightarrow 0^{+}} \frac{F(x-h)-{ }_{g} F(x)}{(-h)}=F_{g H}(x, 1) .
$$

So

$$
\lim _{h \rightarrow 0} \frac{F(x+h)-{ }_{g} F(x)}{h}=F_{g H}(x, 1) .
$$

Thus, by Definition 2.2, we know that $F(x)$ is $g H$-differentiable at $x$, and

$$
F^{\prime}(x)=F_{g H}(x, 1) .
$$

Sufficiency.

If $F(x)$ is $g H$-differentiable at $x$, then there exists $\delta>0$ such that $x+h \in(a, b), x-h \in(a, b)$, for any $h \in(0, \delta)$, and $F^{\prime}(x) \in[R]$ such that

$$
\lim _{h \rightarrow 0^{+}} \frac{F(x+h)-{ }_{g} F(x)}{h}=\lim _{h \rightarrow 0^{+}} \frac{F(x+h)-{ }_{g} F(x)}{h}=F^{\prime}(x) .
$$

Therefore, $F_{g H}^{+}(x, 1)=F_{g H}^{-}(x, 1)=F^{\prime}(x, 1)$. So the $g H$-derivative of $F(x)$ in direction $y=1$ at $x$ exists, and $F^{\prime}(x)=F_{g H}(x, 1)$. 
Denote $e_{i}=\left(a_{1}, a_{2}, \ldots, a_{j}, \ldots, a_{n}\right)(i=1,2, \ldots, n)$ with

$$
a_{j}=\left\{\begin{array}{ll}
1 & j=i, \\
0 & j \neq i,
\end{array} \quad(i=1,2, \ldots, n) .\right.
$$

Theorem 3.6. Let $F: M \rightarrow\left([R], d_{H}\right)$ be an interval-valued function $F(x)=[\underline{F}(x), \bar{F}(x)] . x_{0}=$ $\left(x_{1}^{(0)}, x_{2}^{(0)}, \ldots, x_{n}^{(0)}\right) \in M$. If $F(x)$ is $g H$-differentiable in the direction $e_{i}$ at $x_{0}$, then $F(x)$ has the partial $g H$-derivative at $x_{0}$ for $x_{i}$, and $\left(\partial F / \partial x_{i}\right)\left(x_{0}\right)=F_{g H}\left(x_{0}, e_{i}\right)$.

Proof. Let $h_{i}\left(x_{i}\right)=F\left(x_{1}^{(0)}, \ldots, x_{(i-1)}^{(0)}, x_{i}, x_{(i+1)}^{(0)}, x_{n}^{(0)}\right)$, then

$$
\begin{aligned}
& \frac{h_{i}\left(x_{i}+h\right)-{ }_{g} h_{i}\left(x_{i}\right)}{h}=\frac{F\left(x_{0}+h e_{i}\right)-{ }_{g} F\left(x_{0}\right)}{h}, \\
& \frac{h_{i}\left(x_{i}\right)-{ }_{g} h_{i}\left(x_{i}-h\right)}{h}=\frac{F\left(x_{0}\right)-{ }_{g} F\left(x_{0}-h e_{i}\right)}{h} .
\end{aligned}
$$

Since the $g H$-derivative of $F(x)$ in direction $e_{i}$ at $x_{0}$ exists, i.e., there exists $F_{g H}\left(x_{0}, e_{i}\right) \in[R]$ such that

$$
\lim _{h \rightarrow 0^{+}} \frac{F\left(x_{0}+h e_{i}\right)-{ }_{g} F\left(x_{0}\right)}{h}=\lim _{h \rightarrow 0^{+}} \frac{F\left(x_{0}\right)-{ }_{g} F\left(x_{0}-h e_{i}\right)}{h}=F_{g H}\left(x_{0}, e_{i}\right) .
$$

Therefore, we have

$$
\lim _{h \rightarrow 0^{+}} \frac{h_{i}\left(x_{i}+h\right)-{ }_{g} h_{i}\left(x_{i}\right)}{h}=\lim _{h \rightarrow 0^{+}} \frac{h_{i}\left(x_{i}\right)-{ }_{g} h_{i}\left(x_{i}-h\right)}{h}=F_{g H}\left(x_{0}, e_{i}\right) .
$$

By Theorem 3.5, we have $h_{i}\left(x_{i}\right)$ is $g H$-differentiable at $x_{i}^{(0)}$. So by Definition 2.4, we can know that $F(x)$ has the partial $g H$-derivative at $x_{0}$ for $x_{i}$, and $\left(\partial F / \partial x_{i}\right)\left(x_{0}\right)=F_{g H}\left(x_{0}, e_{i}\right)$.

Corollary 3.7. Let $F: M \rightarrow[R]$ be an interval-valued function $F(x)=[\underline{F}(x), \bar{F}(x)] . \quad x_{0}=$ $\left(x_{1}^{(0)}, x_{2}^{(0)}, \ldots, x_{n}^{(0)}\right) \in M$. If the $g H$-derivatives of $F(x)$ exist on some neighborhoods of $x_{0}$ in the direction $e_{i}(i=1,2, \ldots, n)$ and are continuous at $x_{0}$, then $F(x)$ is $g H$-differentiable at $x_{0}$, and $\nabla F\left(x_{0}\right)=\left\{F_{g H}\left(x_{0}, e_{1}\right), F_{g H}\left(x_{0}, e_{2}\right), \ldots, F_{g H}\left(x_{0}, e_{n}\right)\right\}$.

Proof. Since $F_{g H}\left(x_{0}, e_{i}\right)$ of $F(x)$ exists on some neighborhoods of $x_{0}$ and is continuous at $x_{0}$, by Theorem 3.6, we can know that all $\partial F /\left.\partial x_{i}\right|_{x=x_{0}}(i=1,2, \ldots, n)$ exist on some neighborhoods of $x_{0}$ and

$$
\left(\partial F / \partial x_{i}\right)\left(x_{0}\right)=F_{g H}\left(x_{0}, e_{i}\right)(i=1,2, \ldots, n)
$$

are continuous at $x_{0}$.

Therefore, by Definition 2.5 we can know that $F(x)$ is $g H$-differentiable at $x_{0}$ and

$$
\nabla F\left(x_{0}\right)=\left\{F_{g H}\left(x_{0}, e_{1}\right), F_{g H}\left(x_{0}, e_{2}\right), \ldots, F_{g H}\left(x_{0}, e_{n}\right)\right\} .
$$

\section{Acknowledgment}

This work is supported by the National Science Foundation of China (11461052) and the Inner Mongolia Natural Science Foundation of China (2014MS0107). 


\section{References}

[1] B. Bede, L. Stefanini, Generalized differentiability of fuzzy-valued functions, Fuzzy Sets and Systems, 230 (2013), 119141. 1

[2] Y. Chalco-Cano, H. Roman-Flores, M. D. Jimenez-Gamero, Generalized derivative and $\pi$-derivative for set-valued functions, Inform. Sci., 181 (2011), 2177-2188. 1

[3] Y. Chalco-Cano, A. Rufián-Lizan, H. Román-Flores, M. D. Jiménez-Gamero, Calculus for intervalvalued functions using generalized Hukuhara derivative and applications, Fuzzy Sets and Systems, 219 (2013), 49-67. 1

[4] V. Lupulescu, Fractional calculus for interval-valued functions, Fuzzy Sets and Systems, 265 (2015), 63-85. 1. 2.1, 2.2

[5] R. E. Moore, Interval Analysis,Prentice-Hall, Englewood Cliffs, New Jersey, (1966). 1

[6] R. Osuna-Gómez, Y. Chalco-Cano, B. Hernández-Jiménez, G. Ruiz-Garzn, Optimality conditions for generalized differentiable interval-valued functions, Inform Sci., 321 (2015), 136-146. 1, 2.2, 2.4, 2.5, 3

[7] L. Stefanini, B. Bede, Generalized Hukuhara differentiability of interval-valued functions and interval differential equations, Nonlinear Anal., 71 (2009), 1311-1328. 1 . 2.3

[8] H.-C. Wu, The KarushKuhnTucker optimality conditions in multiobjective programming problems with interval-valued objective functions, European J. Oper. Res., 196 (2009), 49-60. 1 